\title{
The Ubiquity of Sidon sets that are not $I_{0}$
}

\author{
Kathryn E. Hare and L. Thomas Ramsey
}

\begin{abstract}
We prove that every infinite, discrete abelian group admits a pair of $I_{0}$ sets whose union is not $I_{0}$. In particular, this implies that every such group contains a Sidon set that is not $I_{0}$.
\end{abstract}

\section{Introduction}

A subset $E$ of a discrete abelian group $\Gamma$ with compact dual group $G$ is said to be a Sidon set if every bounded $E$-sequence can be interpolated by the Fourier transform of a measure on $G$. If the measure can be chosen to be discrete, $E$ is called an $I_{0}$ set. Finite sets in any group $\Gamma$ and Hadamard sequences of integers are examples of these interpolation sets in the group $\Gamma=\mathbb{Z}$.

Clearly, $I_{0}$ sets are Sidon. However, the converse is not true since the class of Sidon sets is known to be closed under finite unions, but the class of $I_{0}$ sets is not. Indeed, in 12, Méla gave an example of a pair of Hadamard sets in $\mathbb{Z}$ whose union is not $I_{0}$.

In this note we prove that every infinite, discrete abelian group admits a pair of $I_{0}$ sets whose union is not $I_{0}$. Consequently, every such group admits a Sidon set that is not $I_{0}$. Our method is constructive and establishes even more: We prove that given any infinite subset $F \subseteq \Gamma$ there are $I_{0}$ sets $E \subseteq F$ and $E^{\prime} \subseteq F+F-F$, whose union is not $I_{0}$ (but is, of course, Sidon). In fact, we show that the sets $E, E^{\prime}$ have stronger interpolation properties than just $I_{0}$. These depend upon the algebraic properties of the initial set $F$.

\section{Preliminaries}

Let $G$ be a compact abelian group and $\Gamma$ its discrete abelian dual group. One example is $G=\mathbb{T}$, the circle group of complex numbers of modulus one, with dual group $\Gamma=\mathbb{Z}$.

Definition 1. (i) A subset $E \subseteq \Gamma$ is said to be Sidon if for every bounded function $\phi: E \rightarrow \mathbb{C}$ there is a measure $\mu$ on $G$ with $\widehat{\mu}(\gamma)=\phi(\gamma)$ for all $\gamma \in E$. If the interpolating measure $\mu$ can always be chosen to be discrete, then the set $E$ is said to be $I_{0}$.

2000 Mathematics Subject Classification. Primary 43A46.

Key words and phrases. Sidon set, $I_{0}$ set, Kronecker set.

This research is supported in part by NSERC \#44597.

This paper is in final form and no version of it will be submitted for publication elsewhere. 
(ii) A subset $E \subseteq \Gamma$ is said to be $\varepsilon$-Kronecker if for every $\phi: E \rightarrow \mathbb{T}$ there exists $x \in G$ such that

$$
|\phi(\gamma)-\gamma(x)|<\varepsilon \text { for all } \gamma \in E
$$

and is called weak $\varepsilon-$ Kronecker if the strict inequality is replaced by $\leq$.

Hadamard sets $E=\left\{n_{j}\right\} \subseteq \mathbb{N}$ with Hadamard ratio $q=\inf n_{j+1} / n_{j}>1$ are $I_{0}$ sets [11, thus both $\left\{3^{j}\right\}_{j \geq 1}$ and $\left\{3^{j}+j\right\}_{j \geq 1}$ are $I_{0}$ subsets of $\mathbb{Z}$. However, Méla, in [12, proved that their union is not $I_{0}$. This shows that not all Sidon sets in $\mathbb{Z}$ are $I_{0}$ since it is a deep result, first obtained by Drury [1, with a later proof given by Pisier [13, that any finite union of Sidon sets (in any group $\Gamma$ ) is Sidon. It is an open problem whether every Sidon set is a finite union of $I_{0}$ sets.

Hadarmard sets with ratio $q>2$ are also known to be weak $\varepsilon$-Kronecker with $\varepsilon=\left|1-e^{i \pi(q-1)}\right|$. In fact, every $\varepsilon$-Kronecker set is $I_{0}$ if $\varepsilon<\sqrt{2}[\mathbf{5}$ and is Sidon if $\varepsilon<2[\mathbf{8}$.

It is known that every infinite subset of $\Gamma$ contains infinite $I_{0}$ sets and if $\Gamma$ does not contain any elements of order 2 , then every infinite subset contains an infinite weak 1-Kronecker set; c.f., [4, [6], 7] and the references cited there. Of course, if $\Gamma$ consists of only elements of order two, then it does not contain any $\varepsilon$-Kronecker set with $\varepsilon<\sqrt{2}$.

The main result of this paper is to show that every infinite, discrete abelian group admits a pair of $I_{0}$ sets that are weak $\varepsilon$-Kronecker for suitable $\varepsilon$, but whose union is not $I_{0}$. The number $\varepsilon$ can always be chosen to be at most $\sqrt{2}$ and often can be taken to be arbitrarily small, such as when $\Gamma$ is torsion free. As far as we are aware, this is the first proof that every infinite, discrete abelian group admits Sidon sets that are not $I_{0}$. Knowing the existence of such sets is useful, for instance, in studying the space of weakly almost periodic functions on $\Gamma$; c.f., [3. p. 17], as well as [2] for further background.

Like Méla's original proof, our argument is constructive and relies upon the well known Hartman/Ryll-Nardzewski characterization of $I_{0}$ sets in terms of the Bohr topology on $\bar{\Gamma}$, the Bohr compactification of $\Gamma$.

Proposition 1. 9] A subset $E \subseteq \Gamma$ is $I_{0}$ if and only if whenever $E_{1}$ and $E_{2}$ are disjoint subsets of $E$, then $E_{1}$ and $E_{2}$ have disjoint closures in $\bar{\Gamma}$.

With this, one can quickly prove the following generalization of Méla's argument.

Lemma 1. Suppose that 0 is a cluster point of $\left\{\chi_{n}\right\}_{n=1}^{\infty} \subseteq \Gamma$ in the Bohr compactification of $\Gamma$. If the sets $E=\left\{\gamma_{n}\right\} \subseteq \Gamma$ and $E^{\prime}=\left\{\gamma_{n}+\chi_{n}\right\} \subseteq \Gamma$ are disjoint, then $E \cup E^{\prime}$ is not an $I_{0}$ set.

Proof. Assume the subnet $\left\{\chi_{n_{\alpha}}\right\}$ (indexed by $\alpha$ ) converges to 0 in $\bar{\Gamma}$. Since $\bar{\Gamma}$ is compact, there is a subnet (not relabelled) such that $\left\{\gamma_{n_{\alpha}}\right\}$ converges to some $\gamma \in \bar{\Gamma}$. Since $\left\{\chi_{n_{\alpha}}\right\}$ also converges to 0 along this subnet, it follows that $\left\{\gamma_{n_{\alpha}}+\chi_{n_{\alpha}}\right\}$ converges to $\gamma$. But that means the disjoint sets $E$ and $E^{\prime}$ both have $\gamma$ in their closures. By Proposition 1 their union is not $I_{0}$.

In the case of $\Gamma=\mathbb{Z}$, one can take $\left\{\chi_{n}\right\}=\mathbb{N}$. For the more general situation, we will take $\left\{\chi_{n}\right\}=\bigcup_{m=1}^{\infty} H_{m}$, where the sets $H_{m}$ are constructed in the following lemma. 
LEMMA 2. If $F$ is any infinite subset of $\Gamma$, then there is a countable subset $H \subseteq(F-F) \backslash\{0\}$ which has 0 as a cluster point in the Bohr topology. Indeed, we can take $H=\bigcup_{m=1}^{\infty} H_{m}$ where for each positive integer $m, H_{m}$ is a finite subset of $(F-F) \backslash\{0\}$ having the property that for all $x_{1}, \ldots, x_{m} \in G$ there is some $\gamma \in H_{m}$ with

$$
\sup _{1 \leq j \leq m}\left|\gamma\left(x_{j}\right)-1\right|<\frac{1}{m} .
$$

Proof. To begin, we claim that there is some $\phi \in \bar{\Gamma}$ with the property that if $V$ is an open neighbourhood of $\phi$, then $V \cap F$ is infinite. If not, then for every $\phi \in \bar{\Gamma}$ there is an open neighbourhood of $\phi$, say $U_{\phi}$, such that $U_{\phi} \cap F$ is finite. As the open sets $U_{\phi}$ cover $\bar{\Gamma}$ and $\bar{\Gamma}$ is compact, we can choose a finite subcover, $\left\{U_{\phi_{j}}\right\}_{j=1}^{J}$. But that contradicts the assumption that $F$ is infinite and hence proves the claim.

In particular, if $x_{1}, \ldots, x_{m} \in G$, then

$$
V=\left\{\gamma \in \bar{\Gamma}: \sup _{j=1, \ldots, m}\left|\gamma\left(x_{j}\right)-\phi\left(x_{j}\right)\right|<\frac{1}{2 m}\right\}
$$

is a neighbourhood of $\phi$ and hence must contain infinitely many elements from $F$. For each such collection $x_{1}, \ldots, x_{m}$, choose $f_{1} \neq f_{2}$ in $F$ such that $\left|f_{i}\left(x_{j}\right)-\phi\left(x_{j}\right)\right|<$ $1 /(2 m)$ for all $j=1, \ldots, m$. Then $\left|f_{1}\left(x_{j}\right)-f_{2}\left(x_{j}\right)\right|<1 / m$ for $i=1,2$ and all $j=1, \ldots, m$. Since $f_{1}-f_{2} \in F-F \subseteq \Gamma$, and is therefore a continuous character on $G$, there is a neighbourhood of $\left(x_{1}, \ldots, x_{m}\right) \in G^{m}$, denoted $W\left(f_{1}, f_{2}\right)$, such that if $\left(y_{1}, \ldots, y_{m}\right) \in W\left(f_{1}, f_{2}\right)$, then

$$
\sup _{j=1, \ldots, m}\left|f_{1}\left(y_{j}\right)-f_{2}\left(y_{j}\right)\right|<\frac{1}{m} .
$$

As $G^{m}$ is compact, there are finitely many such neighbourhoods, $W\left(f_{1}^{(k)}, f_{2}^{(k)}\right)$, for $k=1, \ldots, N_{m}$, so that $G^{m}=\bigcup_{k=1}^{N_{m}} W\left(f_{1}^{(k)}, f_{2}^{(k)}\right)$. The set

$$
H_{m}=\left\{f_{2}^{(k)}-f_{1}^{(k)}: k=1, \ldots, N_{m}\right\}
$$

meets the requirements of the lemma.

The observation that $H=\bigcup_{m=1}^{\infty} H_{m}$ clusters at 0 follows directly from the fact that any neighbourhood of 0 in the Bohr topology contains a subset of the form $\left\{\gamma: \sup _{j=1, \ldots, m}\left|\gamma\left(x_{j}\right)-1\right|<1 / m\right\}$ for some positive integer $m$ and $x_{1}, \ldots, x_{m} \in$ $G$.

Before turning to the details of the proof of our main result, we list some other elementary facts about Kronecker and $I_{0}$ sets which can be found in [7] and will be used in the proof of our main result.

LEMmA 3. (i) Suppose $\pi: \Gamma \rightarrow \Lambda$ is a homomorphism that is an injection on $E \subseteq \Gamma$. If $\pi(E)$ is weak $\varepsilon$-Kronecker (or $I_{0}$ ) as a subset of the group $\pi(\Gamma)$, then $E$ is weak $\varepsilon$-Kronecker (resp., $I_{0}$ ) as a subset of $\Gamma$.

(ii) Suppose $\Lambda$ is a subgroup of $\Gamma$ and that $E$ is weak $\varepsilon$-Kronecker (or $I_{0}$ ) as a subset of the group $\Lambda$. Then $E$ is also weak $\varepsilon$-Kronecker (resp., $I_{0}$ ) as a subset of $\Gamma$. 


\section{The Main Result}

3.1. Statement and outline of the proof. Here is the statement of our main result.

THEOREM 1. If $F$ is any infinite subset of a discrete abelian group $\Gamma$, there are countable, disjoint sets $E \subseteq F$ and $E^{\prime} \subseteq F+F-F$ such that both $E$ and $E^{\prime}$ are $I_{0}$, but $E \cup E^{\prime}$ is not $I_{0}$. Furthermore, the sets $E, E^{\prime}$ can be chosen to be weak $\varepsilon$-Kronecker for suitable $\varepsilon \leq \sqrt{2}$.

REMARK 1. The choice of $\varepsilon$ will be clear from the proof and depends on algebraic properties of $F$. As will be seen in the proof, in many situations $\varepsilon$ can be chosen to be arbitrarily small.

Since the union of any two Sidon sets is again Sidon, we immediately obtain the following corollary.

Corollary 1. Every infinite, discrete abelian group admits a Sidon set that is not $I_{0}$.

The remainder of the paper will be devoted to proving the theorem. Its proof depends upon the general structure theory for abelian groups.

Theorem 2. (see [7, p. 165]) Given any discrete abelian group $\Gamma$, there is an index set $\mathcal{I}$ such that $\Gamma$ is isomorphic to a subgroup of

$$
\Omega=\bigoplus_{\alpha \in \mathcal{I}} \Omega_{\alpha}
$$

where for each $\alpha$ either $\Omega_{\alpha}=\mathbb{Q}$ or there is a prime number $p_{\alpha}$ such that $\Omega_{\alpha}=$ $C\left(p_{\alpha}^{\infty}\right)$, the group of all $p_{\alpha}^{n}$-th roots of unity.

Throughout the remainder of the paper $\pi_{\alpha}$ will denote the projection from $\Omega$ onto the factor group $\Omega_{\alpha}$. Our proof of Theorem [1 will be constructive and will depend on the following two cases:

Case 1: There is some index $\alpha \in \mathcal{I}$ such that $\pi_{\alpha}(F)$ is infinite. This case will be handled by Lemma 4 when $\Omega_{\alpha}=\mathbb{Q}$ and Lemma 5 when $\Omega_{\alpha}=C\left(p_{\alpha}^{\infty}\right)$. We will see that we can even arrange for the sets $E$ and $E^{\prime}$ to be $\varepsilon$-Kronecker for any given $\varepsilon>0$. Making the choice with $\varepsilon<\sqrt{2}$ ensures $E, E^{\prime}$ are both $I_{0}$.

Case 2: Otherwise, $\pi_{\alpha}(F)$ is finite for all indices $\alpha \in \mathcal{I}$ and then there must be an infinite subset $\mathcal{J} \subseteq \mathcal{I}$ such that for each $\alpha \in \mathcal{J}$ there is some $\lambda \in F$ with $\pi_{\alpha}(\lambda) \neq 0$. The existence of such an infinite subset of indices allows us to either construct sets $E, E^{\prime}$ that are weak $\varepsilon$-Kronecker for some $\varepsilon<\sqrt{2}$ (and hence $I_{0}$ ) or to construct sets $E, E^{\prime}$ that are both independent (and hence $I_{0}$ ) and weak $\sqrt{2}$-Kronecker. The choice of construction depends on the orders of the non-zero characters $\pi_{\alpha}(\lambda)$. This argument can be found in Lemma 6 .

In both cases, the two sets we construct will be disjoint and have the form $E=\left\{\gamma_{n}\right\}, E^{\prime}=\left\{\gamma_{n}+\chi_{n}\right\}$ where $\left\{\chi_{n}\right\}$ clusters at 0 . Thus the fact that $E \cup E^{\prime}$ is not $I_{0}$ will follow immediately from Lemma 1

We now turn to handling these two cases.

\subsection{Proof of the Theorem in Case 1 .}

Lemma 4. Suppose there exists an index $\alpha \in \mathcal{I}$ such that $\pi_{\alpha}(F)$ is infinite and $\Omega_{\alpha}=\mathbb{Q}$. Given any $\varepsilon>0$, there are infinite disjoint sets $E \subset F$ and $E^{\prime} \subset F+F-F$ such that $E$ and $E^{\prime}$ are weak $\varepsilon$-Kronecker and $I_{0}$, but $E \cup E^{\prime}$ is not $I_{0}$. 
Proof. Let $H=\bigcup_{m=1}^{\infty} H_{m}=\left\{\chi_{n}\right\}_{n=1}^{\infty} \subseteq(F-F) \backslash\{0\}$ be a set that clusters at 0 , constructed in Lemma 2 Fix $0<\varepsilon<\sqrt{2}$ and assume we can find a sequence of characters $\lambda_{n} \in \pi_{\alpha}(F)$ such that:

(a) $V=\left\{\lambda_{n}\right\}$ and $V^{\prime}=\left\{\lambda_{n}+\pi_{\alpha}\left(\chi_{n}\right)\right\}$ are weak $\varepsilon$-Kronecker sets in $\Omega_{\alpha}$; and

(b) For $n \neq n^{\prime}$ we have $\lambda_{n} \neq \lambda_{n^{\prime}}, \lambda_{n} \neq \lambda_{n^{\prime}}+\pi_{a}\left(\chi_{n^{\prime}}\right)$, and $\lambda_{n}+\pi_{\alpha}\left(\chi_{n}\right) \neq$ $\lambda_{n^{\prime}}+\pi_{a}\left(\chi_{n^{\prime}}\right)$.

Then, for each $\lambda_{n}$ choose some $\gamma_{n} \in F$ such that $\pi_{\alpha}\left(\gamma_{n}\right)=\lambda_{n}$. Set $E=\left\{\gamma_{n}\right\} \subseteq$ $F$ and $E^{\prime}=\left\{\gamma_{n}+\chi_{n}\right\} \subseteq F+F-F$. By construction, $\pi_{\alpha}$ is one-to-one from $E$ to $V$ and one-to-one from $E^{\prime}$ to $V^{\prime}$. Condition (b) and the fact that $H$ consists of nonzero characters implies that $E$ and $E^{\prime}$ consist of distinct terms and are disjoint.

By Lemma 3(i), $E$ and $E^{\prime}$ inherit the weak $\varepsilon$-Kronecker property from $V$ and $V^{\prime}$ respectively. Since $\varepsilon<\sqrt{2}$, both $E$ and $E^{\prime}$ are $I_{0}$. Furthermore, because 0 is a cluster point of the set $H$, Lemma 1 implies that $E \cup E^{\prime}$ is not $I_{0}$.

Thus the proof of the lemma will be complete if we can construct a sequence of characters satisfying the two conditions (a) and (b). This will be an induction argument which depends on whether $\pi_{\alpha}(F)$ is a subset of a group isomorphic to $\mathbb{Z}$ or it is not.

First, suppose that there is some integer bound $B>0$ such that for all $\lambda \in$ $\pi_{\alpha}(F)$, there are are integers $b>0$ and $a$ such that $\pi_{\alpha}(\lambda)=a / b$ and $b \leq B$. Then $\pi_{\alpha}(F)$ is a subset of the (additive) subgroup $\frac{1}{B !} \mathbb{Z}$ of $\mathbb{Q}$. Because $H \subseteq F-F$, we also have $\pi_{\alpha}(H)$ contained in this subgroup.

Given $\varepsilon>0$, choose an integer $q>2$ such that $\pi /(q-1)<\varepsilon$. As $\pi_{\alpha}(F)$ is infinite, we may inductively choose $\lambda_{n} \in \pi_{\alpha}(F)$, sufficiently large in modulus, so that both $V$ and $V^{\prime}$ are Hadamard sequences in $\mathbb{Q}$ with Hadamard ratio $\geq q$ and condition (b) is satisfied. By [7, Prop. 2.2.6], $V$ and $V^{\prime}$ are both weak $\varepsilon$-Kronecker subsets of $\frac{1}{B !} \mathbb{Z}$ and by Lemma 3 (ii) they are also both weak $\varepsilon$-Kronecker sets in $\Omega_{\alpha}$. Thus condition (a) is satisfied.

Otherwise, for every positive integer $B$, there is some $s / t \in \pi_{\alpha}(F)$ with $t>B$ and $\operatorname{gcd}(s, t)=1$. (We will say $s / t$ is in reduced form.) In this case we need to carefully account for the denominators of rational numbers. Note that any $x \in \mathbb{Q}$ has a unique reduced form, $s / t$, and we will write $D(x)$ for the denominator $t$.

Given $\varepsilon>0$, choose an integer $q>2$ such that $\pi / q<\varepsilon$. Let $B_{0}=D\left(\pi_{\alpha}\left(\chi_{1}\right)\right)$ and choose $\lambda_{1} \in \pi_{\alpha}(F)$ such that $D\left(\lambda_{1}\right)>q B_{0}$ !. Assuming $\lambda_{1}, \ldots, \lambda_{n}$ have been inductively constructed for $n \geq 1$, let

$$
B_{n}=2 \max \left\{D\left(\lambda_{i}\right), D\left(\pi_{\alpha}\left(\chi_{j}\right)\right): 1 \leq i \leq n, 1 \leq j \leq n+1\right\} .
$$

Now choose $\lambda_{n+1} \in \pi_{\alpha}(F)$ so that $D\left(\lambda_{n+1}\right)>q B_{n}$ !. This choice ensures that $\lambda_{i}$ and $\lambda_{i}+\pi_{\alpha}\left(\chi_{i}\right)$ for $1 \leq i \leq n$, as well as $\pi_{\alpha}\left(\chi_{n+1}\right)$, all belong to $\frac{1}{B_{n} !} \mathbb{Z}$, while $\lambda_{n+1}$ and $\lambda_{n+1}+\pi_{\alpha}\left(\chi_{n+1}\right)$ are outside $\frac{1}{B_{n} !} \mathbb{Z}$. It follows that condition (b) will be satisfied for $V$ and $V^{\prime}$.

We argue next that $V^{\prime}$ is $\varepsilon$-Kronecker in $\Omega_{\alpha}$. To this end, let $\phi: V^{\prime} \rightarrow \mathbb{T}$, say $\phi\left(\lambda_{n}+\pi_{\alpha}\left(\chi_{n}\right)\right)=t_{n} \in \mathbb{T}$. We need to prove there is some character $g \in \widehat{\mathbb{Q}}$, the dual of $\mathbb{Q}$, such that

$$
\left|g\left(\lambda_{n}+\pi_{\alpha}\left(\chi_{n}\right)\right)-t_{n}\right|<\varepsilon \text { for all } n .
$$

As explained in $[10,25.5]$, elements of $\widehat{\mathbb{Q}}$ can be identified with sequences $\left\{\omega_{n}\right\} \subset \mathbb{T}$, subject to the constraints that $\omega_{n+1}^{n+1}=\omega_{n}$, with the understanding that $g(1 / n !)=$ 
$\omega_{n}$. Clearly it will be sufficient to satisfy the consistency condition

$$
\omega_{B_{n+1}}^{B_{n+1} ! / B_{n} !}=\omega_{B_{n}}
$$

provided that for $j \notin\left\{B_{n}\right\}$, say $B_{n}<j<B_{n+1}$, one specifies

$$
\omega_{j}=\omega_{B_{n+1}}^{B_{n+1} ! / j !} \text {. }
$$

To start the specification of $g$, set $\omega_{B_{0}}=1$. This ensures that if $k \in \mathbb{Z}$, then $g(k)=\omega_{B_{0}}^{k B_{0}}$ ! hence $g(\mathbb{Z})=1$. (This will be helpful in the next lemma as it allows us to interpret $g$ as a character on $\mathbb{Q} / \mathbb{Z}$.) Since $\pi_{\alpha}\left(\chi_{1}\right)=s / B_{0}$ ! for some integer $s$, it follows that

$$
g\left(\pi_{\alpha}\left(\chi_{1}\right)\right)=\omega_{B_{0}}^{s}=1 .
$$

By Equation (3.2) one may choose $\omega_{B_{1}}$ to be any $J$-th root of unity, where $J=$ $B_{1} ! / B_{0}$ !, in other words, one can choose any integer $K \in[0, J-1]$ and specify

$$
\omega_{B_{1}}=e^{2 \pi i K / J} .
$$

Because $\lambda_{1} \in \frac{1}{B_{1} !} \mathbb{Z}$, the reduced form of $\lambda_{1}$ is $s / t$ with $t$ dividing $B_{1}$ !. Thus, with $y / z$ the reduced form of $B_{0} ! / t$, we have

$$
g\left(\lambda_{1}\right)=\left(e^{2 \pi i K / J}\right)^{s B_{1} ! / t}=e^{2 \pi i K s B_{0} ! / t}=e^{2 \pi i K s y / z} .
$$

Since $t=D\left(\lambda_{1}\right)>q B_{0} !$, the reduced form of $B_{0} ! / t$ is $y / z$ with $z>q$. Both $s$ and $y$ are relatively prime to $z$, hence the exponential $e^{2 \pi i s y / z}$ is a primitive $z$-th root of unity. By definition, $B_{1} \geq 2 t$ and $t>q B_{0}$ !, thus $J=B_{1} ! / B_{0} ! \geq B_{1}>t \geq z$. This means we can choose any of the $z$-th roots of unity as the value for $g\left(\lambda_{1}\right)$. If we make a choice that is closest to $t_{1}$, then the angular difference between $g\left(\lambda_{1}\right)$ and $t_{1}$ is at most $\pi / z$ and thus

$$
\left|g\left(\lambda_{1}+\pi_{\alpha}\left(\chi_{1}\right)\right)-1\right|=\left|g\left(\lambda_{1}\right)-t_{1}\right|<\frac{\pi}{z}<\frac{\pi}{q}<\varepsilon .
$$

We proceed to define $\left\{\omega_{B_{n}}\right\}$ inductively. Assume that for $1 \leq j \leq n$ we have specified $\omega_{B_{j}}$ so that $\left|g\left(\lambda_{j}+\pi_{\alpha}\left(\chi_{j}\right)\right)-t_{j}\right|<\varepsilon$. By the definition of $B_{n}$ we know that $\pi_{\alpha}\left(\chi_{n+1}\right)$ is in $\frac{1}{B_{n} !} \mathbb{Z}$. Therefore $g$ has already been specified at $\pi_{\alpha}\left(\chi_{n+1}\right)$. However, $\lambda_{n+1} \notin \frac{1}{B_{n} !} \mathbb{Z}$, but is inside $\frac{1}{B_{n+1} !} \mathbb{Z}$, so the selection of $\omega_{B_{n+1}}$ will determine $g\left(\lambda_{n+1}\right)$.

By Equation (3.2), $\omega_{B_{n+1}}$ can be chosen to be any $J$-th root of $\omega_{B_{n}}$, where $J=B_{n+1} ! / B_{n}$ !. If we write $e^{i \theta}$ for $\omega_{B_{n}}$, then we are free to choose any integer $K \in[0, J-1]$ and define

$$
\omega_{B_{n+1}}=e^{i \theta / J} \cdot e^{2 \pi i K / J} .
$$

Let $s / t$ be the reduced form of $\lambda_{n+1}$ and $y / z$ the reduced form for $B_{n} ! / t$. Since $t$ divides $B_{n+1}$ !, we have

$$
g\left(\lambda_{n+1}\right)=\omega_{B_{n+1}}^{s B_{n+1} ! / t}=e^{i \theta s B_{n} ! / t} \cdot e^{2 \pi i K s B_{n} ! / t}=e^{i \theta s B_{n} ! / t} \cdot e^{2 \pi i K s y / z} .
$$

As before we see that any of the $z$-th roots of unity can be used to help define $g\left(\lambda_{n+1}\right)$ and we make the choice (of $K$ ) so that the corresponding $z$-th root of unity differs in angle by at most $\pi / q$ from

$$
\left(e^{i \theta s B_{n} ! / t}\right)^{-1} g\left(\pi_{\alpha}\left(\chi_{n+1}\right)\right)^{-1} t_{n+1} .
$$


(We remind the reader that the first two factors above are known as they have already been determined by $\omega_{B_{n}}$.) Therefore

$$
\begin{aligned}
\left|g\left(\lambda_{n+1}+\pi_{\alpha}\left(\chi_{n+1}\right)\right)-t_{n+1}\right| & =\left|e^{2 \pi i K s y / z}-\left(e^{i \theta s B_{n} ! / t}\right)^{-1} g\left(\pi_{\alpha} \chi_{n+1}\right)^{-1} t_{n+1}\right| \\
& <\frac{\pi}{z}<\frac{\pi}{q}<\epsilon .
\end{aligned}
$$

Since the choice of $g$ satisfies (3.1), it follows that $V^{\prime}$ is $\varepsilon$-Kronecker.

The proof that $V$ is $\varepsilon$-Kronecker is similar, but easier, as the factors $g\left(\pi_{\alpha}\left(\chi_{n+1}\right)\right)$ are not present. This shows that condition (a) also holds and that completes the proof of the Lemma.

Lemma 5. Suppose there exists an index $\alpha \in \mathcal{I}$ such that $\pi_{\alpha}(F)$ is infinite and $\Omega_{\alpha}=C\left(p^{\infty}\right)$. Given any $\varepsilon>0$, there are infinite disjoint sets $E \subset F$ and $E^{\prime} \subset F+F-F$ such that $E, E^{\prime}$ are weak $\varepsilon$-Kronecker and $I_{0}$, but $E \cup E^{\prime}$ is not $I_{0}$.

Proof. Let $H=\left\{\chi_{n}\right\} \subseteq(F-F) \backslash\{0\}$ be a countable set that clusters at 0 , as in the previous lemma. We identify $C\left(p^{\infty}\right)$ with a subgroup of $\mathbb{Q} / \mathbb{Z}$, so that for every $\lambda$ in the subgroup generated by $F$ there is some $x_{\lambda} \in \mathbb{Q}$ such that $\pi_{\alpha}(\lambda)=x_{\lambda}+\mathbb{Z}$.

Because $\pi_{\alpha}(F)$ is infinite, the set of minimal denominators $\left\{D\left(x_{\lambda}\right): \lambda \in F\right\}$ must be unbounded. The proof of the second part of Lemma 4 shows that given $0<\varepsilon<\sqrt{2}$ there is a sequence $V=\left\{x_{\lambda_{n}}\right\}$ such that both $V$ and $V^{\prime}=\left\{x_{\lambda_{n}}+x_{\chi_{n}}\right\}$ are $\varepsilon$-Kronecker sets in $\mathbb{Q}$, with the interpolation being done by characters $g \in \widehat{\mathbb{Q}}$ such that $g(\mathbb{Z})=1$, and hence by characters on $\mathbb{Q} / \mathbb{Z}$. These can also be viewed as characters on $C\left(p^{\infty}\right)$ if the domain is suitably restricted. Of course, $g\left(\pi_{\alpha}\left(\lambda_{n}\right)\right)=$ $g\left(x_{\lambda_{n}}+\mathbb{Z}\right)=g\left(x_{\lambda_{n}}\right)$ and $g\left(\pi_{\alpha}\left(\lambda_{n}+\chi_{n}\right)\right)=g\left(x_{\lambda_{n}}+x_{\chi_{n}}\right)$. It follows that both $\left\{\pi_{\alpha}\left(\lambda_{n}\right)\right\}$ and $\left\{\pi_{\alpha}\left(\lambda_{n}+\chi_{n}\right)\right\}$ are $\varepsilon$-Kronecker subsets of $\Omega_{\alpha}$.

The construction in the proof of the previous lemma also ensures that the pullbacks, $E=\left\{\lambda_{n}\right\}$ and $E^{\prime}=\left\{\lambda_{n}+\chi_{n}\right\}$, are disjoint, have distinct terms and are $\varepsilon$-Kronecker in $\Gamma$. That their union is not $I_{0}$ follows immediately from Lemma 1.

REMARK 2. We note that similar arguments can be used to prove that under the assumption that $\pi_{\alpha}(F)$ is infinite for some $\alpha$ there are infinite sets, $E, E^{\prime}$, that are $I_{0}$ and have the property that for each $\varepsilon>0$ the sets $E$ and $E^{\prime}$ contain cofinite subsets that are $\varepsilon$-Kronecker and whose union is not $I_{0}$. The latter statement is a consequence of the fact that the union of an $I_{0}$ set and a finite set is known to be $I_{0}$ (see [7, p.63]).

\subsection{Proof of the Theorem in Case 2 .}

Lemma 6. Suppose $\pi_{\alpha}(F)$ is finite for all $\alpha \in \mathcal{I}$, but that $\mathcal{I}_{q}$ is infinite for some $q \geq 2$, where

$$
\mathcal{I}_{q}=\left\{\alpha \in \mathcal{I}: \exists \lambda \in F \text { s.t. } \pi_{\alpha}(\lambda) \text { has order at least } q\right\} .
$$

Let $|\exp (i \pi / q)-1|=\varepsilon_{q}$. There are infinite disjoint sets $E \subset F$ and $E^{\prime} \subset F+F-F$ such that $E$ and $E^{\prime}$ are both weak $\varepsilon_{q}$-Kronecker and $I_{0}$, but $E \cup E^{\prime}$ is not $I_{0}$.

Proof. Again, let $H=\left\{\chi_{n}\right\}_{n=1}^{\infty} \subseteq(F-F) \backslash\{0\}$ be a countable set that clusters at 0 . 
First, assume $\mathcal{I}_{q}$ is infinite for some $q \geq 3$. Let $\beta_{1} \in \mathcal{I}$ be chosen with the property that $\pi_{\beta_{1}}\left(\chi_{1}\right)=0$ and there is some $\lambda_{1} \in F$ with $\pi_{\beta_{1}}\left(\lambda_{1}\right)$ having order $\geq q$. We can do this since there are only finitely many indices $\beta$ with $\pi_{\beta}\left(\chi_{1}\right) \neq 0$. Now inductively choose $\beta_{n} \in \mathcal{I}$ and $\lambda_{n} \in F$ such that $\pi_{\beta_{n}}\left(\lambda_{n}\right)$ has order at least $q$,

$$
\pi_{\beta_{n}}\left(\chi_{m}\right)=0 \text { for all } m \leq n \text { and } \pi_{\beta_{n}}\left(\lambda_{m}\right)=0 \text { for all } m<n \text {. }
$$

Set $\Pi$ equal to the projection from $\Omega$ onto $\Lambda=\bigoplus \Omega_{\beta_{n}}$. Then $\left\{\Pi\left(\lambda_{n}\right)\right\}$ and $\left\{\Pi\left(\lambda_{n}+\right.\right.$ $\left.\left.\chi_{n}\right)\right\}$ are sequences of distinct elements of $\Lambda$ such that $\Pi\left(\lambda_{m}\right) \neq \Pi\left(\lambda_{n}+\chi_{n}\right)$ if $m \neq n$. Since $\chi_{n} \neq 0$, the sets $E=\left\{\lambda_{n}\right\}$ and $E^{\prime}=\left\{\lambda_{n}+\chi_{n}\right\}$ are disjoint. Moreover $\Pi\left(\lambda_{n}+\chi_{n}\right)=\pi_{\beta_{n}}\left(\lambda_{n}\right)+\rho_{n}$ where $\pi_{\beta_{k}}\left(\rho_{n}\right)=0$ for all $k \geq n$.

We claim that $\left\{\Pi\left(\lambda_{n}+\chi_{n}\right)\right\}$ is weak $\varepsilon_{q}$-Kronecker. To prove this, note that a character $g$ on $\Lambda$ is specified as $g=\left\{g_{n}\right\}$, with each $g_{n}$ a character on $\Omega_{\beta_{n}}$. Let $M_{n}$ be the order of $\pi_{\beta_{n}}\left(\lambda_{n}\right)$. For any $M_{n}$-th root of unity, $\omega_{n}$, there is a character $g_{n}$ such that $g_{n}\left(\pi_{\beta_{n}}\left(\lambda_{n}\right)\right)=\omega_{n}$. Thus given $\left\{t_{n}\right\} \subseteq \mathbb{T}$, we may inductively specify $g_{n}$ so that

$$
\left|g\left(\pi_{\beta_{n}}\left(\lambda_{n}\right)\right)-g\left(\rho_{n}\right)^{-1} t_{n}\right| \leq\left|\exp \left(i \pi / M_{n}\right)-1\right| \leq \varepsilon_{q} .
$$

Thus

$$
\left|g\left(\Pi\left(\lambda_{n}+\chi_{n}\right)\right)-t_{n}\right|=\left|g\left(\pi_{\beta_{n}}\left(\lambda_{n}\right)\right) g\left(\rho_{n}\right)-t_{n}\right| \leq \varepsilon_{q},
$$

which proves the claim.

The argument that $\left\{\Pi\left(\lambda_{n}\right)\right\}$ is weak $\varepsilon_{q}$-Kronecker is similar. By Lemma 3 , $E$ and $E^{\prime}$ are also weak $\varepsilon_{q}$-Kronecker. As $q \geq 3$, we have $\varepsilon_{q} \leq 1$ and hence $E$ and $E^{\prime}$ are $I_{0}$. As before, their union is not $I_{0}$.

Otherwise, we can assume $\mathcal{I}_{2}$, but not $\mathcal{I}_{3}$, is infinite. Then there is a finite (possibly empty) set $\mathcal{J} \subseteq \mathcal{I}$ such that for all $\lambda \in F$ and all $\alpha \in \mathcal{I} \backslash \mathcal{J}$, either $\pi_{\alpha}(\lambda)=0$ or has order 2 . Repeat the construction as above, but this time with the additional requirement that $\beta_{n} \in \mathcal{I} \backslash \mathcal{J}$. As before, the sets $E, E^{\prime}$ that arise from the construction are disjoint and they are both weak $\sqrt{2}$-Kronecker. Moreover, $\Pi(E)$ and $\Pi\left(E^{\prime}\right)$ are both independent sets of elements of order 2 and one can easily verify that such sets are $I_{0}$ (c.f. [7, p. 66]). It follows from Lemma 3 that $E$ and $E^{\prime}$ are both $I_{0}$ sets, while their union is not.

These three lemmas complete the proof of the main theorem since the assumption that $F$ is infinite guarantees that either $\pi_{\alpha}(F)$ is infinite for some $\alpha$, or there are infinitely many indices $\alpha$ with $\pi_{\alpha}(F)$ not trivial and in that case $\mathcal{I}_{q}$ is infinite for some $q \geq 2$.

\section{References}

[1] S. Drury, Sur les ensembles de Sidon, C.R. Acad. Sci. Paris 271A(1970), 162-163.

[2] M. Filali and J. Galindo, Approximable WAP and LUC-interpolation sets, Adv. in Math. 233(2013), 87-114.

[3] M. Filali and J. Galindo, Interpolation sets and the size of quotients of function spaces on a locally compact group, preprint 2016.

[4] J. Galindo and S. Hernandez, The concept of boundedness and the Bohr compactification of a MAP abelian group, Fund. Math. 15(1999), 195-218.

[5] C.C. Graham and K.E. Hare, $\varepsilon$-Kronecker and $I_{0}$ sets in abelian groups, I: arithmetic properties of $\varepsilon$-Kronecker sets, Math. Proc. Camb. Philo. Soc. 140(2006), 475-489.

[6] C.C. Graham and K.E. Hare, Existence of large $\varepsilon$-Kronecker sets and $F Z I_{0}(U)$ sets in discrete abelian groups, Colloq Math. 127(2012), 1-15.

[7] C.C. Graham and K.E. Hare, Interpolation and Sidon Sets for Compact Groups, SpringerVerlag, New York, 2013. 
[8] K.E. Hare and L.T. Ramsey, The relationship between $\varepsilon-$ Kronecker and Sidon sets, to appear Can. Math. Bull.

[9] S. Hartman and C. Ryll-Nardzewski, Almost periodic extensions of functions, Colloq. Math. 12(1964), 23-39.

[10] E. Hewitt and K.A. Ross, Abstract harmonic analysis, volume I, Springer-Verlag, New York, 1979.

[11] K. Kunen and W. Rudin, Lacunarity and the Bohr topology, Math. Proc. Camb. Phil. Soc. 126(1999), 117-137.

[12] J.-F. Méla, Sur les ensembles d'interpolation de C. Ryll-Nardzewski et de S. Hartman, Studia Math. (1968), 168-193.

[13] G. Pisier, Conditions d'entropie et caracterisations arithmetique des ensembles de Sidon. In Proc. Conf. on modern topics in harmonic analysis, Inst. de Alta Math, Torino, 1982, 911-941.

Dept. of Pure Mathematics, University of Waterloo, Waterloo, Ontario, Canada N2L 3G1

E-mail address: kehare@uwaterloo.ca

Dept. of Mathematics, University of Hawait, Honolulu, Hi., USA 96822

E-mail address: ramsey@math.hawaii.edu 